\title{
Proceso de formación profesional y consumo musical
}

\section{Process of professional formation and musical consumption}

\author{
Hermila Loya Chávez ${ }^{1}$ \\ Juan Tenorio Urbina ${ }^{2}$ \\ José Antonio Ávila Quevedo 3
}

\section{Resumen}

Constituye un reporte parcial de una investigación de enfoque fenomenológico y de tipo descriptiva cuyo propósito es identificar y analizar el consumo musical de los estudiantes universitarios del área de educación, como un referente de su realidad sociocultural y transformación educativa en el contexto de una universidad pública. El reporte presenta los contenidos generales de las letras de las canciones que escuchan con más frecuencia, la forma en que lo hacen y sus razones, todo ello en la temporalidad de la investigación que es el año 2017. Así mismo se encontraron relaciones con el desarrollo de la formación profesional en el área de educación. Para la obtención de datos se generó y aplicó una encuesta y se realizaron indagaciones en grupos focales integrados por los estudiantes de la carrera de licenciatura en intervención educativa. En relación al contenido y su análisis se encontró que existe una disonancia

\footnotetext{
${ }^{1}$ Hermila Loya Chávez. Profesora de la Universidad Pedagógica Nacional del Estado de Chihuahua, México. Es Doctora en Educación e integrante del Cuerpo Académico Formación, Identidad y Comunicación. Correo electrónico: loyahermila@gmail.com ID: http://orcid.org/0000-0001-6420-1061

2 Juan Tenorio Urbina. Maestro de tiempo completo en la Universidad Pedagógica Nacional del Estado de Chihuahua, Campus Chihuahua, México. Miembro del Cuerpo Académico en Formación "Formación docente, identidad y comunicación". Correo electrónico: jtenorio@upnech.edu.mx ID: http://orcid.org/0000-0002-0298-351X

${ }^{3}$ José Antonio Ávila Quevedo. Asesor académico de la Universidad Pedagógica Nacional del Estado de Chihuahua, Campus Cuauhtémoc, México. Integrante del CA en Formación UPN-CA- 95. Correo electrónico: javila@upnech.edu.mx

ID: http://orcid.org/0000-0001-6613-881X
} 
cultural entre los mensajes de estas canciones y los ideales que se marcan desde la visión humanista de la sociedad que fundamenta su carrera profesional.

\title{
Palabras clave
}

Consumo, música, formación profesional, análisis del discurso.

\begin{abstract}
It constitutes a partial report of a phenomenological approach of descriptive type, whose purpose is to identify and analyze the musical consumption of university students in the area of education, as a reference of their sociocultural reality and educational transformation in the context of a public university. The report presents the general contents of the lyrics of the songs they listen to the most, the way they do it and their reasons, all in the temporality of the investigation that is the year 2017. Likewise, there were relations with the development of professional training in the area of education. In order to obtain data, survey was generated and applied and inquiries were made in focus groups composed of the students of the degree program in educational intervention.
\end{abstract}

\section{Keywords}

Consumption, music, professional training, discourse analysis.

\section{Contenido}

Esta investigación analiza el consumo musical de los alumnos universitarios como medio para explorar su realidad sociocultural influenciada por las modas alternantes que hacen la diversidad de rasgos culturales de la sociedad hoy día.

La música es uno de los elementos de mayor consumo cultural por parte de los estudiantes debido a la facilidad de acceso que tienen mediante los dispositivos electrónicos de orden personal que les permiten seleccionar la variedad de producciones, géneros, intérpretes y videos en el tiempo y espacio que ellos mismos determinan.

Desde la línea antropológica cultural, García Canclini (1993) plantea que el consumo cultural es el conjunto de procesos de apropiación y uso de productos en los que el valor simbólico es más importante que los valores de cambio y de uso. La práctica obedece a una construcción y atribución simbólica que van haciendo los sujetos. 
Para Geertz (1988) el consumo se deriva del sistema de símbolos en los que los miembros de la sociedad desarrollan tramas de significación que se interpretan de manera diferente por cada miembro. Bermúdez (2001) plantea que el consumo cultural es un proceso en el cual los actores sociales se apropian y hacen circular los objetos atendiendo a su valor simbólico abriendo sentido a sus relaciones y formando sus identidades.

La atribución del valor simbólico a los consumos culturales según Bermúdez (2001) se genera en la trama de las relaciones intersubjetivas entre los sujetos. En este proceso se expresan las necesidades de ser del individuo y de su búsqueda y consolidación de identidad en la que persigue una forma de ser y una distinción para ser admitido y agrupado socialmente con sus características. Bourdieu (1990) establece que el consumo integra símbolos, ideas y valores que conforman un habitus que generan la aprehensión del mundo del sujeto que se concreta en sus prácticas sociales.

Martín- Barbero (2003) desde la dimensión de la comunicación humana establece que el consumo musical consiste en la apropiación de las audiencias de los productos, junto con el sistema que permite la apropiación relaciones, significados y asignación de sentidos. Desde esta perspectiva los usos sociales del producto definen el desarrollo del consumo cultural.

Por otro lado, en las universidades no se considera el origen pluricultural de los estudiantes pues la oferta universitaria son los programas curriculares y culturales propios. Así el perfil socio-cultural de los estudiantes se va desarrollando en su realidad en la que el consumo musical constituye un elemento de significado para la construcción de la imagen personal y profesional.

El consumo musical es un elemento de la educación informal, cultura con la cual ingresan los jóvenes a la universidad. Esta cultura musical es sólo una parte de su cultura total. Para ellos no representa problema porque constituye una forma de disfrutar las diferentes expresiones, sin embargo la expresión de las culturas revelan formas de pensamiento de los jóvenes de las sociedades actuales que pueden conllevar una reproducción del orden social, económico, político y cultural que implica el refuerzo o la resistencia al planteamiento curricular para su formación profesional.

El consumo musical que hacen los estudiantes de área de educación interesa por la repercusión que tiene en su formación y en su posterior ejercicio profesional, una vez que pueden convertirse en promotores de consumos que afecten el desarrollo de los individuos o grupos que atienden. Por ello es importante que en la formación de un profesional de la educación se analice este fenómeno ya que tiene implicaciones en el sentido, la interpretación y en la reproducción de la práctica del consumo. 
Los estudiantes son sensibles a la expresión musical y desarrollan identidad con los nuevos géneros que reflejan una visión de la vida no precisamente coherente con los propósitos educativos, misma que puede impactar en su formación, mediando en una imagen profesional no contemplada en el modelo de formación. Así el consumo es uno de los vectores relevantes a la hora de plantear el proceso de formación de los profesionales de la educación y una forma de diagnosticar este vector es a través del análisis de los contenidos de la letra de las canciones que les gusta escuchar.

Esta investigación responde a la pregunta ¿Qué características tiene el consumo musical de los estudiantes de la licenciatura en intervención educativa? Con el objetivo general de analizar las características del consumo musical en cuanto a contenido de las letras y la forma en que se da ese consumo. Así mismo analizar los contenidos de la letra de las canciones en boga y su relación con la formación de la imagen profesional que asumen como futuros interventores educativos.

\section{Método}

Es una investigación de corte fenomenológico. Como enfoque que busca conocer la conciencia del mundo y no al mundo mismo Bolio (2012). La ruta de la investigación y de recaudo de datos fue: primero una encuesta exploratoria de 64 preguntas organizadas en siete secciones, con preguntas de respuesta abierta y cerrada, dirigido a estudiantes de segundo y octavo semestres de la Licenciatura en Intervención Educativa de nueve regiones del estado de Chihuahua con el propósito de integrar un cuerpo de datos de los estudiantes de la LIE acerca del consumo musical. Como método de validación del contenido del cuestionario para la obtención de datos se procedió a la técnica de jueceo (Escobar y Cuervo, 2008) en la cual participaron tres expertos. La población sobre la cual se trabajó en con la encuesta fueron 367 estudiantes como población total de la Licenciatura en Intervención Educativa y adscritos a nueve de los diez campus de la Universidad.

A través del análisis del discurso se revisa el contenido de las canciones que los estudiantes escuchan con más frecuencia para identificar marcos culturales reflejados en sus contenidos e identificar sus características. El análisis crítico del discurso según Van Dijk (1999) establece al discurso como una práctica social que analiza el uso del lenguaje más allá de la frase, de la acción y de la interacción y por tanto del discurso, en términos de las estructuras, procesos e imposiciones sociales, políticas, culturales e históricas. Con este fundamento se realizó un 
detallado y sistemático análisis de las estructuras y estrategias de los contenidos de la letra en sus relaciones con los contextos sociales y políticos: relaciones de poder (económicas, políticas y de género) considerando que la relación de poder en la estructura social se refleja en el discurso como una herramienta comunicativa y por ende en todas sus relaciones se reflejan estas.

Dos grupos focales constituidos por estudiantes que se encuentran en la parte final de la carrera. Fueron seleccionados por informantes clave bajo criterios. El propósito fue que aportaran datos acerca de géneros y canciones que escuchan, así como las explicaciones del por qué lo hacen. Esta técnica según Kitzinger (1995) consiste en un espacio de opinión para captar el sentir, pensar y vivir de los individuos, mediante la provocación de autoexplicaciones para obtener datos cualitativos.

Una vez obtenida la información se realizó un análisis cruzando los resultados del grupo focal con los resultados del análisis del contenido de las canciones para determinar hasta qué punto el análisis del consumo musical sirve como referente de los procesos que se están desarrollando en la formación profesional de los LIE.

\section{Resultados}

El reguetón es el género más escuchado por los estudiantes de la Licenciatura en Intervención Educativa. Lo escuchan en casi todos los lugares y ten cualquier tiempo gracias a los dispositivos electrónicos. Es común que lo escuchen en la escuela, cuando abordan el autobús o el automóvil, en las reuniones de amigos, en la calle, en los bares, en los antros, en los restaurantes, en los establecimientos comerciales y en las oficinas y en los centros de trabajo.

Los estudiantes escuchan reguetón en sus reuniones para buscar una pareja, para atraer a alguien mediante el movimiento de sus cuerpos. También dicen que les gusta esta música por su ritmo, para mover su cuerpo sensualmente, así mismo reconocen que algunas letras denigran al ser humano, pero que lo bailan una vez que han ingerido alcohol y sustancias que se sirven comúnmente en las reuniones.

Prefieren el reguetón porque los alegra por el ritmo rápido y pegajoso, en contraste a los ritmos lentos que conducen a emociones de melancolía y tristeza. El reguetón les ayuda a entrar en confianza con otros jóvenes, el participante I3 dice "Si no conoces a alguien, pues bailando, te haces muy compa", es decir cada uno se relaja y se facilita incluirse a los grupos. En este sentido, el participante I5 plantea "me fijo en el ritmo de la música, no en el contenido de la letra, esa sale sobrando, realmente es el 
ritmo el que te atrae". Reconocen que en la letra, por un lado, se denigra a la mujer y que por otro sirve para elevar su autoestima al bailar y llamar la atención de los presentes, el I6 dice que "el reguetón hace sentir el centro de la atención, que es deseada y que vale como mujer". En términos de recepción musical los estudiantes escuchan el reguetón solos o acompañados. Depende de la circunstancia en que se encuentran y el contexto y que los sitios son todo su espacio pues están pletóricos de música, en todo lugar y momento. Los dispositivos les permiten hacerlo. Se comparten siempre los sitios electrónicos y las novedades en You tube y en Spotify. Han dejado atrás dispositivos como el Ipad, el casset y el disco compacto. Usan generalmente teléfonos de tercera y cuarta generación. También prefieren escuchar con audífonos.

Cabe aclarar que se escucha menos el radio porque no permite la selección de música. Esta generación de jóvenes se caracteriza por el uso de listas de reproducción. Así lo señala I11" brincamos de un cantante a otro sin necesidad de escuchar todo su disco".

Los grupos o cantantes que escuchan: Maluma, Ozuna, Bad Bunny, Ezequiel, Daddy Yankee, Becky G, Carolina Rosse, Christian Nogal, Julion Alvarez, Remmy Valenzuela, Los perdidos de Sinaloa, El fantasma, Shakira, Jessy Joe, Sin bandera, Río Roma, Luis Fonsi, Farruco, Enrique Iglesias, Cuatro Bally, Miranda, Calle 13, J. Balvin, Willy William, Imagen dragon, Katy Perry, Cartel de Santa, Yandell, Demmy Lobato, Banda MS, Oldies como Queens, Chicago, Michael Jackson, entre otros citados.

El acto de escuchar música preferentemente lo realizan solos o con amigos. Los estudiantes prefieren su "propio play" y que lo pueden alternar, modificar, apagar, volver a prender. Algunas de las canciones que actualmente les gustan a los estudiantes son del género reguetón, pop, rap, banda, sierreña, norteña, cumbias y otros, se presentan en la Tabla 1.

Tabla 1

Títulos de canciones que les gustan a los estudiantes LIE

\begin{tabular}{|l|l|}
\hline Despacito & Luis Fonsi / Ozuna/ daddy Yankee \\
\hline Shaky Shaky: & Daddy Yankee \\
\hline Que se canse de llamar: & Carolina Ross/ Los plebes del rancho \\
\hline 3 AM & Jessy \& Joe / Gente de Zona \\
\hline Se preparó & Ozuna \\
\hline La rompecorazones & Ozuna/ Daddy Yankee \\
\hline Me reclama & Ozuna/ Luigi 21 \\
\hline
\end{tabular}




\begin{tabular}{|l|l|}
\hline Becky G. y Bad Bunny & Mayores \\
\hline Me enamoré de ti & Remmy Valenzuela \\
\hline No me hubiera enamorado & Cornelio Vega \\
\hline Crippy Kush & Bad Bunny, Farruko \\
\hline Adios amor & Cristian Nodal \\
\hline Criminal & Ozuna/ Natti Natasha \\
\hline Mi buen amor & Enrique Bunbury/ Mom Laferte \\
\hline Me rehuso & Danny Osean \\
\hline Felices los cuatro & Maluma \\
\hline Escápate conmigo & Ozuna, Wisin \\
\hline Te fallé & Cristian Nodal \\
\hline Loco enamorado & Remmy Valenzuela \\
\hline Perfecta & Miranda \\
\hline Atrévete te te & Calle 13 \\
\hline Una Lady como tu & Manuel Turizo/ Nicky Jam \\
\hline Mi gente & J Balvin y Willy William \\
\hline Tu foto & \\
\hline Thunder & Ozuna \\
\hline Swit & Imagen dragon \\
\hline Bailame & Katy Perry \\
\hline Sorry, dont sorry & Yandell \\
\hline
\end{tabular}

Los contenidos de letra que ellos prefieren plantean la alegría, la inclusión, el amor, la fraternidad, el respeto, la convivencia, la solidaridad, por ejemplo la canción Mi gente de J. Balvin y Willy Williams, algunas canciones de Ozuna como "Tu foto", otras de Karla Morris como "Eres tú", canciones de Mom Laferte como "Mi buen Amor", del grupo Los perdidos de Sinaloa "Si tú quieres" es decir que no propicien estados de ánimo negativos.

\section{Discusión}

Considerando los ejes del análisis del discurso para abordar las letras de las canciones preferidas por los estudiantes: Hegemonía, poder, dominio, ideología, clase, género y discriminación.

Poder. Considerando que el poder constituye un ejercicio que produce sus formas mediante una transformación de los individuos e indica como fabrica las relaciones de sometimiento concretos que se constituyen en las formas simbólicas. García Canclini (1993) plantea que un producto cultural se caracteriza por el valor simbólico, que este implica el manejo de 
estructuras por el consumidor que le permiten reconocerlas. En las letras se encuentra la ligereza del acto de consumo de drogas, de alcohol, de actividades no legales para conseguir dinero, el uso de armas por personas jóvenes, las relaciones de pareja furtivas, la evitación del compromiso, la carencia de dinero como algo natural, la vida al día (finanzas, salud, seguridad), la discriminación de la mujer, el amor como mercancía y la preponderancia de los temas juveniles en los temas que se abordan. Hay un proceso de identificación del estudiante con determinada letra y música que ocurre cuando se logra el manejo de esas estructuras dentro de su espacio sociocultural en desarrollo.

La clase social. Se ha encontrado una profunda identidad entre los que se agrupan como iguales. En los contenidos de las canciones es recurrente la alusión a sus estados de clase en los que se advierte el reconocimiento y adhesión a ese estado. Las estructuras simbólicas adquieren sentido para el sujeto estudiante en medida que relacionan los constructos simbólicos presentes en él y formando una construcción dialéctica de nuevos significados a partir de la recepción, la cual se da de manera rizómica ante la nueva información, según lo plantean Deleuze y Guattari (1997).

Género. Dos aspectos, el primero, hay preponderancia de la autoría de la letra por parte de los hombres, así mismo la interpretación es mayor por parte de ellos, en este conjunto de letras que se han analizado. El segundo, la relación de pareja se define en términos del acto sexual para ambos.

No obstante, el problema radica en que la población participante en la investigación, que son el aproximadamente el $80 \%$ mujeres, son quienes prefieren y hacen suyas las letras de las canciones, por lo cual son las consumidoras directas que aceptan el tema y el contenido. Bajtin (2000) precisa que los significados se estructuran en forma de narraciones por parte del sujeto y Lizarazo ( 1998) que el sujeto va interrelacionando en formas de constelaciones diegéticas hasta llegar a constituir la asunción específica del entorno cultural por parte del individuo, y a partir del cual se construye la subjetividad del mismo.

Hegemonía. La visión de mundo de los jóvenes consumidores no deviene de manera determinada por el contenido de las canciones, sino por la construcción de significados de los sujetos en su realidad. Se encontró interés en temas que cuestionan al gobierno, al poder, a la policía, a toda aquella entidad que los limite a vivir la libertad en todos los sentidos, entonces, la música, como producto cultural es apreciada por los sujetos a 
partir de la posibilidad de ligarla con otros productos y su acceso a ellos desde su posibilidad de decodificación y encodificación de la estructura simbólica.

Así también a los estudiantes les gustan los contenidos temáticos de los procesos sociales, sus problemas como la política, la corrupción, las traiciones, el narcotráfico, en los que se encuentre la protesta y la exposición de estas.

El consumo musical refleja la estructura diegética del sujeto, es decir la construcción de su estructura significativa, que funciona como un filtro que delimita los mensajes posibles de recibir, y por otro lado marca las significaciones aceptables para el sujeto que le permitan asimilar significados relevantes y, por tanto, les permite discriminar que productos culturales forman parte de su gusto.

\section{Conclusiones}

En relación al objetivo general de esta investigación se ha encontrado que en el proceso inicial de los estudiantes disfrutan la música sin poner atención a la letra, ellos escuchan lo que los demás y se dejan llevar generalmente por el ritmo, que les permita sentirse alegres y dinámicos, proceso que va transformándose según avanzan en los semestres de su carrera.

Aunque los estudiantes proceden de diferentes localidades y estas constituyen diferentes regiones en el estado, mismas en las que prevalecen diferentes características sociales, culturales y económicas, algunos de los géneros que escuchan son constantes por ejemplo el reguetón se encuentra en preferencia de todos los estudiantes, el género sierreño es igualmente aceptado no importando si sus vidas las desarrollan en la ciudad o en el medio rural. La música de banda es muy aceptada sobre todo en los conciertos y en las fiestas y reuniones. Los dispositivos móviles son los que han permitido la generalización de las preferencias, permitiendo que se identifiquen con todos estos géneros.

Conforme a lo esperado en esta investigación se pudo encontrar dos elementos contradictorios: El primero es que sus preferencias musicales se van transformando con el proceso de formación en la carrera. Los estudiantes proponen los y las cantantes menos agresivas y que no incorporan temas de fuerte contenido de violencia, de actividad ilegal, entre otras. Se considera que los contenidos de formación en la carrera son altamente críticos de la realidad y que los estudiantes, una vez que han cursado cinco semestres, poseen una gran cantidad de herramientas para discriminar lo que escuchan y hacen solo para ellos. Y la segunda situación 
es que aún impera una situación en los estudiantes: ellos dicen que, por un lado, seguirán escuchando esta música en las reuniones, en las fiestas, en el trabajo, inclusive que la compartirán pero no alcanzan a verse en los compromisos sociales que ya están adquiriendo con su carrera. Es decir, ellos dicen que en este momento no prescinden de la música que escuchan pero que si les corresponde trabajo de campo, ya sea de prácticas profesionales o de otros proyectos, entonces estarían pendientes del tipo de música que seleccionarían para trabajar con los individuos o los grupos.

El hallazgo más importante consiste en el proceso de formación contribuye para que los estudiantes de la licenciatura en intervención educativa vayan discriminando los contenidos de las letras de las canciones.

Se concluye que la forma de recepción de los productos culturales tiene un doble efecto, por un lado se presenta un proceso de identificación con el producto cultural, lo cual genera el gusto, por tanto delimita las posibilidades de consumo, por otra parte, los mensajes que tienen los productos culturales, entendidos como elementos simbólicos que proyectan los autores o intérpretes de estas expresiones, entran en relación dialéctica con el universo simbólico del sujeto, lo cual marca la posibilidad de interpretación del producto cultural, determinando al sujeto a partir de la asunción de los contenidos simbólicos del producto cultural.

El consumo musical forma parte del dispositivo de formación dado el proceso que se describe al plantear que permite la identificación con los mensajes trasmitidos por un lado, y por otro la modificación de la subjetividad del individuo.

\section{Referencias}

Bermúdez, E. (2001). Consumo cultural y representación de identidades juveniles. Consultado en http://lasa.international.pitt.edu/lasa2001/bermudezemilia.pdf

Bajtin, M.M.(1999). Estética de la creación verbal. Consultado en ehttps://filologiaunlp.files.wordpress.com/2012/01/bajtc3adnestc3a9ticade-la-creacion-verbal.pdf

Bolio, A. (2012), Hussler y la fenomenología fundamental: Perspectivas del sujeto en el Siglo XXI. Consultado en http://www.redalyc.org/pdf/340/34024824004.pdf

Bourdieu, P. (1990). Sociología y Cultura. México. Grijalbo. Google libros. http://revintsociologia.revistas.csic.es/index.php/revintsociologia/article/ viewFile/547/569

Deleuze, G. y Guattari, f. (1977). Rizoma: Introducción. Valencia: Pre- Textos. 
Escobar, J. y Cuervo, A. (2008). Validez de contenido y juicio de expertos: Una aproximción a su utilización. Consultado en http://www.redalyc.org/pdf/340/34024824004.pdf

García Canclini, N. (1993). El consumo cultural en México. Consejo Nacional para la Cultura y las Artes. Colección Pensar la Cultura. México.

Geertz, C. (1988). La interpretación de las culturas. Consultado en http://www.colmich.edu.mx/relaciones25/files/revistas/038/Geert zClifford.pdf

Kitzinger, J. (1995) Qualitative research: Introducing focus groups. British Medical Journal, 311, 299-302. doi:10.1136/bmj.311.7000.299

Lisarazo, D. (1998). La reconstrucción del significado. México. Wesley y Longman

Martín-Barbero, J. (2003). Retos culturales de la comunicación a la educación: Elementos para una reflexión que está por comenzar. Comunicación, Medios y Educación: Un Debate para la Educación en Democracia, 44, 19-32.

Van Dijk, T. (1999). El análisis crítico del discurso. Barcelona: Anthropos. 
RECIE. Revista Electrónica Científica de Investigación Educativa Vol. 4, núm. 2, enero-diciembre 2019, pp. 1107-1117. 